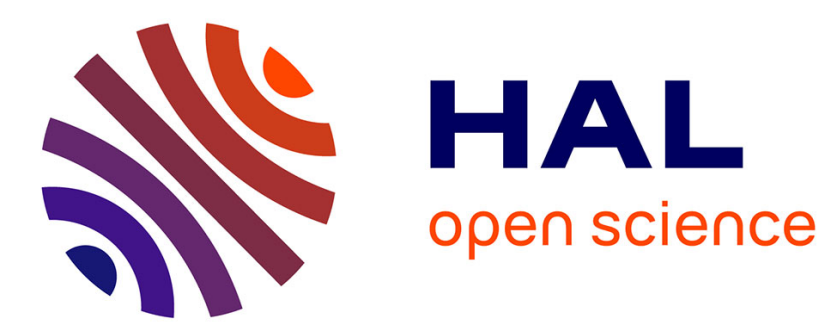

\title{
Les morphèmes de futur en arabe et en berbère. Réflexions pour une typologie \\ Catherine Taine-Cheikh
}

\section{To cite this version:}

Catherine Taine-Cheikh. Les morphèmes de futur en arabe et en berbère. Réflexions pour une typologie. Faits de langues, 2009, 33, pp.91-102. halshs-00564265

\section{HAL Id: halshs-00564265 https://shs.hal.science/halshs-00564265}

Submitted on 10 Feb 2011

HAL is a multi-disciplinary open access archive for the deposit and dissemination of scientific research documents, whether they are published or not. The documents may come from teaching and research institutions in France or abroad, or from public or private research centers.
L'archive ouverte pluridisciplinaire HAL, est destinée au dépôt et à la diffusion de documents scientifiques de niveau recherche, publiés ou non, émanant des établissements d'enseignement et de recherche français ou étrangers, des laboratoires publics ou privés. 


\title{
Les morphèmes de futur en arabe et en berbère Réflexions pour une typologie
}

\author{
Catherine Taine-Cheikh
}

En Afrique du Nord et au Sahara, les parlers arabes et berbères sont en contact depuis des siècles. Ceci peut éclairer certains traits de ressemblances. Cependant, par ailleurs, l'appartenance commune à l'ensemble des langues chamitosémitiques (ou afro-asiatiques), explique une grande similitude sur divers points du système. L'organisation du système verbal autour de la notion d'aspect apparaît comme l'une des caractéristiques de cette famille de langues:

«Dans les systèmes verbaux des langues chamito-sémitiques, le temps situé par rapport au moment de l'énonciation n'a pas d'expression morphologique sinon de façon associée ou historiquement secondaire. De manière générale ce qui est indiqué, ce sont des aspects sous lesquels le procès est considéré, soit dans son déroulement, soit comme événement ou bien encore selon qu'il est général ou habituel ou envisagé dans son unicité » (D. Cohen 1988: 24).

Si la notion d'aspect a toujours la prépondérance sur celle de temps comme catégorie grammaticale, un certain nombre de parlers ont développé des moyens particuliers d'expression du temps verbal. Ceci se vérifie notamment pour le futur, même si cette notion ne se présente pas comme une unité bien définie.

Klein, physicien-philosophe, nous invite à distinguer différentes conceptions du temps et à ne pas confondre, par exemple, le présent du temps physique, de « durée nulle » (simple point entre « l'infini du passé et l'infini du futur») et le présent du temps psychologique où se mélangent « un peu du passé récent et un peu de l'avenir proche » et qui « déploie donc une certaine durée en unifiant ce que le temps physique ne cesse de séparer, en retenant provisoirement ce qu'il emporte, en englobant ce qu'il exclut » (2003: 184).

Quel devrait être alors le présent de la temporalité linguistique et, dans le cas qui nous importe plus encore ici, son futur ? Les deux représentations les plus courantes du temps linguistique suggèrent, soit une représentation ternaire selon le schème passé / présent / futur, soit des relations d'antériorité / postériorité par rapport à un troisième terme de référence constitué par le moment de l'énonciation ou un événement particulier. Cette différenciation, qui fait écho aux deux visions du présent précédemment esquissées, renvoie aussi à la distinction entre discours et récit. Mais l'expression linguistique du temps se complique, en particulier pour le futur, du fait de l'attitude du locuteur par rapport au nonréalisé. Pour certains linguistes, le futur «pur» (ou «vrai futur ») sera celui de la prédiction ou de l'action considérée comme certaine. Pour d'autres, le lien du 
modal avec le futur relèverait plus du fonctionnement habituel des langues que de l'exception ( $v$. Gosselin 1996).

À défaut de dégager une conception temporelle unitaire pour l'arabe et pour le berbère - a fortiori pour tout le domaine arabo-berbère - , nous verrons que les étymons des morphèmes de futur présentent d'intéressantes similitudes, en accord avec le fait, souligné par Dahl (1985), que «the meaning signalled by future morphemes is similar cross-linguistically » (Bybee, Pagliuca \& Perkins 1991: 17). Cependant, les parallélismes qu'on peut établir entre les deux groupes de parlers sont limités par l'existence, en berbère, d'une expression indirecte du futur dont on ne trouve pas de véritable équivalent en arabe.

\section{LE MODAL BERBERE A VALEUR DE FUTUR}

Le système verbal repose principalement sur une opposition d'ordre aspectuel entre un accompli et un inaccompli (Galand 1977), et si des indications proprement temporelles du type demain ou il y a deux jours peuvent être présentes dans l'énoncé, le choix de l'aspect suffit le plus souvent à situer l'événement dans le temps par rapport à l'instant T0 de l'énonciation.

Il existe cependant une troisième forme verbale pan-berbère: l'aoriste (AOR). Du point de vue de la valeur, cet aoriste présente des points communs avec l'inaccompli, mais, contrairement à ce dernier, il semble trop indéterminé pour actualiser une forme prédicative autonome. Seul, il apparaît donc surtout en position de dépendance, lorsque le cadre est apporté par un premier verbe (dans une énumération) ou par une première proposition (principale pour une complétive, subordonnée temporelle pour une proposition principale, protase d'une conditionnelle pour son apodose, ...).

La présence de la particule $a d$ (vraisemblablement d'origine déictique) permet toutefois à l'aoriste d'apparaître dans de nouveaux contextes, à la fois en position initiale dans les énoncés simples ou complexes et comme syntagme prédicatif indépendant. Alors que l'aoriste construit un événement dépourvu de tout ancrage situationnel précis, la présence de ad lui confère une actualisation virtuelle sur l'axe référentiel du possible. Cette construction de l'aoriste avec ad, qui sert également à d'autres expressions du modal (pour l'injonctif et l'optatif, v. ex. (1) et (2)) n'est pas spécialisée dans la dénotation du futur (ex. (3)). Elle peut cependant exprimer le futur dans la majorité des dialectes, en le «connotant », selon la distinction de Galand (idem: 302). En tant que futur non factuel, il est exclu de certains contextes (prédicats négatifs et propositions relatives, notamment), ceux-ci requérant - comme cela a été observé dans d'autres langues du monde - des tournures plus assertives.

Tamazight (Bentolila 1981: 140, 147 et 173)

(1) ad ur Gar (IMPER)

(2) ad ax in $\breve{G} a$ (AOR) r $r$ i

"Ne jette pas!"

(3) ad aznx (AOR) iflus

"Que Dieu nous protège!"

"J'enverrai l'argent".1

${ }^{1}$ Chez les Aït Seghrouchen, l'emploi de $d u \check{C} a$ "demain" devant l'aoriste suffit pour situer le procès dans le futur (Bentolila, ibid.: 150). 


\section{DES MODALITES ORIENTEES VERS L'AGENT}

Les verbes de volonté et de désir constituent une famille de «future grams» universellement attestée. Leur emploi est plus fréquent en arabe qu'en berbère.

\subsection{En berbère}

Deux verbes de volonté sont à l'origine d'expressions spécifiques de futur.

a) En chleuh, l'expression du futur ne se fait pas, comme dans beaucoup de parlers, par la construction non spécifique $a d+\mathrm{AOR}$, car

« le chleuh s'est donné une marque du futur, par figement d'un emploi du verbe iry "vouloir": ira^ad "il veut que" est devenu la particule $\mathrm{rad}$, suivie de l'aoriste ou de l'inaccompli [...]. La négation $u r$ est admise devant $\mathrm{rad}$ : on entend [uRad] et [urad].

Ex. rad alsg "je recommencerai", ur^rad alsg "je ne recommencerai pas" » (Galand 1988: 236).

Dans certaines variétés régionales où l'évolution vers le figement est encore en cours, elle se produit d'abord au négatif (Leguil 1982), dans un contexte où $a d+$ AOR n'est pas admis.

b) Dans d'autres parlers, le verbe (a)xs/(a) $\dot{g} s$ "vouloir", suivi de $a d$, fournit des formes particulières de futur. Dans les oasis du Sahara septentrional algérien, l'emploi du verbe auxiliaire traduit une insistance sur la proximité de l'action.

Mzab (Chaker 1997: 117), lit. il-veut ad il-meurt

(4) yexsa ad yemmet $\quad$ "Il est sur le point de mourir, il va mourir"

Ailleurs $^{2}$, le figement de la construction, très prononcé, a abouti à deux séries de variantes: celles comme $x a-a d, x a(d)$ ou $\dot{g} a(d)$ qui ont conservé la radicale vélaire de $x a-a d, x a(d)$ ou $\dot{g} a(d)$ et celles comme $s a-a d$, sad et $s a(d)$ qui ont conservé la radicale sifflante. La nuance apportée semble être de certitude ou de volonté.

Figuig (Kossmann 1997: 270)
(5a) ad i-rah
"Il ira (probablement)"
(5b) s-ad i-ṛạ
"Il ira (sûrement)"

Il arrive que le choix de $s a-đ$ soit contraint. Ainsi à Aklim, dans le Rif oriental, après l'auxiliaire du passé ttugia (v. Kossmann 2000: 137):

(6) ttug sađ ruhag

"J'avais l'intention d'aller"

\footnotetext{
${ }^{2}$ Notamment dans les parlers du Nord-Maroc (Figuig et Rif) et dans quelques parlers orientaux (Néfousa et, autrefois, Djerba, v. Brugnatelli 2005: 139; peut-être Siwa, v. Laoust 1931: 53).
} 
Catherine Taine-Cheikh

\subsection{En arabe}

Divers morphèmes de futur se laissent rapporter à un tout petit nombre d'étymons de sens volitif ou désidératif.

a) Le verbe bag $\bar{a}$ (racine BGंY) "demander, désirer, chercher à avoir" de l'arabe classique a de nombreux correspondants dans les dialectes, sous des formes exceptionnellement variées $(b(a) g \bar{a}, b b \bar{a}$ et $b \bar{a})$. La conjugaison suffixale de l'accompli et celle, préfixale, de l'inaccompli peuvent même avoir une base radicale différente, ainsi baga yabi dans le parler arabique du Najd. Si les deux ont des emplois de futur plus ou moins modal (d'intention ou d'imminence), c'est la forme réduite $(b i$-) de l'inaccompli yabi qui sert pour le « vrai » futur.

Des formes comparables, avec des valeurs d'intention ou de futur, ont été relevées en divers points du Maghreb, mais c'est dans les dialectes du MoyenOrient que la particule de futur $b i-, b a-, b$-, est attestée à la plus grande échelle ${ }^{3}$.

b) Le verbe $r \bar{a} d$ "vouloir" (correspondant du classique Parāda, forme dérivée de RWD) est parfois employé à l'inaccompli pour exprimer le futur. Cela concerne essentiellement les parlers bédouins Sāwi parlés dans le croissant fertile, du Golfe persique jusqu'à Alep (Syrie) et même Urfa (Turquie).

Dialectes bédouins d'Urfa (Procházka 2003: 83)

(7) arīd ašrab "I shall drink" (and "I want to drink")

Au Maghreb, rād paraît inusité, mais le verbe dār qui, à l'origine, a le sens de "tourner autour de", sert parfois à exprimer l'imminence. En effet, dans deux parlers méridionaux, au Tchad et en Mauritanie, dār (ou dawwar la forme dérivée « causative ») semble avoir hérité, à l'inaccompli, du sens volitif de rād. Au plan sémantique, la grammaticalisation est plus ou moins complète selon les parlers ${ }^{4}$, mais aucun figement n'a été signalé pour cet étymon.

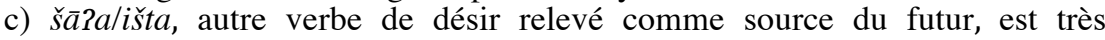
fréquent, mais il n'a guère été relevé en dehors de la Péninsule arabique (hormis quelques attestations de šta et $\breve{s} a t i$ à Constantine, en Algérie).

$\mathrm{Au}$ Yémen, ce verbe subit d'importantes réductions formelles. Les marques les plus fréquentes sont $\check{s} a$ - et $\check{s}-$, plus rarement $\check{s} \bar{u} k$, $\check{s} \bar{l} k$ ou même $k$-/ki-. Dans certains parlers, ce préverbe de futur n'est attesté qu'à la 1SG: ainsi ša- à Sanfā? et $k$-/ $k i$ - dans d'autres parlers ${ }^{5}$.

d) Le syntagme prépositionnel $* b$-wudd- est attesté au Moyen-Orient, sur une aire d'emploi étonnamment continue (pour la région Syrie-Liban-Palestine, $v$. Behnstedt 1997: carte 162). Il offre cependant un exemple intéressant de spécialisation: widd- est employé par les bédouins tandis que badd-/bidd- est en usage chez les sédentaires. Dans certains dialectes, le quasi verbe $* b$-wudd- $(+$ affixe) sert régulièrement pour l'expression du futur.

${ }^{3}$ Parlers, souvent de bédouins, de la Péninsule arabique et de quelques régions proches.

${ }^{4}$ Ainsi le mauritanien idōr ne peut-il être employé, à l'instar du tchadien yadawwar, avec

le verbe "mourir" (verbe dont le sémantisme exclut généralement toute volition).

${ }^{5} k$ - représente la finale de $s \bar{u} k$ et $\breve{s} \bar{k} k$, les 1 SG et 2 SG de $s \breve{a}$ ? à l'accompli (Rossi 1938: 262). 
Les morphèmes de futur en arabe et en berbère

Dialectes bédouins du nord d'Israël (Rosenhouse 1981: 45)

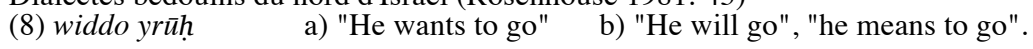

\section{DES VERBES DE MOUVEMENT}

Les verbes de mouvement, parmi lesquels on distingue les verbes du type go et ceux de type come, constituent une seconde famille de «future grams» universellement attestée. Dans le domaine arabo-berbère, ce sont généralement les verbes du premier type qui ont donné de véritables morphèmes de futur.

\subsection{En arabe}

Les dialectes arabes font souvent appel à des formes dérivées des verbes de mouvement, et notamment à leur participe qui « apparaît comme une forme marquée, fortement expressive et permettant de ce fait de souligner éventuellement l'imminence ou le caractère certain d'un procès donné métaphoriquement comme actuel » (Cohen 1984: 271).

a) $m \bar{a} s ̌ i$ et $\dot{g} \bar{a} d i$ sont les formes participiales de deux verbes signifiant "aller, partir". Employés seuls, ces participes indiquent une action non achevée mais déjà commencée (ou considérée comme telle) : "prêt à partir, en train de partir". Suivis d'un verbe à l'inaccompli, ces participes tendent à conférer, comme le verbe français aller, une valeur d'immédiateté ou d'imminence au procès, en particulier dans les dialectes maghrébins. Comme morphème de futur, les participes deviennent souvent invariables en genre et en nombre, mais ils peuvent encore «porter» la négation verbale ma [...] ši, v. (9a). Il arrive cependant que l'invariabilité s'accompagne d'une réduction formelle plus importante : perte du $-i$ final en Tunisie $(m \bar{a} \check{s} i>m \bar{a} \breve{s}$, voire $b \bar{a} \breve{s})$, troncation au Maroc $(m a(a) \breve{s} i>m a$ et surtout $\dot{g} a(a) d i>\dot{g} a)$. Les formes réduites tendent à perdre leur caractère (d'auxiliaire) verbal - d'où le changement de place de l'élément ši en (9b).

Arabe marocain (Ouhalla 2008: 357):

$\begin{array}{llll}\text { (9a) } m a & \dot{g} a d i-\check{s} i & n-m \check{s} i & \text { "I won't go" } \\ \text { (9b) } m a & \dot{g} a & n-m s ̌ i-s ̌ i & \text { "I won't go" }\end{array}$

b) De rāyəh, le participe du verbe rāḥ "partir", dérivent de nombreux morphèmes de futur. En usage dans tout le Moyen-Orient, ils sont généralement invariables et cliticisés. Leur forme très réduite les rendrait souvent impossibles à analyser sans l'existence de variantes libres. Le mono-syllabe $r a \bar{h} h / r a ̣ h$ donne, par altération consonantique de $r / r$, non seulement la variante prévisible $\dot{g} a h(r>\dot{g}$ chez les non musulmans de Baghdad), mais aussi les variantes plus inattendues $l a \bar{h} / l a h$ et yah̆/ya-. La disparition du $r / r$, peut également aboutir à $2 a h$ - et, par métathèse, à ha- (à moins qu'elle ne provienne directement, par tronçation, de la forme féminine raḥa). Si ḥa- est une variante fréquente de la Basse-Égypte et de l'Érythrée, on relève aussi ha- (en Egypte et en Oman), $\{a$ - (dans d'autres régions d'Egypte et dans une partie du Yémen) et même par $? a-$.

c) Dans le domaine maltais, on trouve un autre verbe, synonyme des précédents. La forme participiale sēyyer/seyyer $\left(<*_{s a \bar{P}}\right.$ ir $)$ est employée 
invariablement ou sous une forme plus réduite ( $s \bar{e} r / s e r$ et peut-être $s e-/ s a-)$. Les valeurs propres des différentes variantes semblent difficiles à préciser.

d) $\breve{g} \bar{a} ? / g e(h) / \check{z} a$ "venir" est un des deux seuls verbes de déplacement qui exprime un mouvement centrifuge. Dans quelques parlers où il $\mathrm{y}$ a un début de grammaticalisation (cf. la forme invariable $\breve{g} \bar{a} y$ au sud de l'Iraq), il s'agit d'un véritable morphème de futur. Ailleurs, comme au Caire et en Algérie, l'auxiliaire se conjugue et prend une valeur d'imminence plus que de « vrai » futur.

Djidjelli, Algérie (Marçais 1956: 153)

(10) hœuwwa žăy yeḍ̛rab "Il allait frapper"

e) Le cas de garrab "s'approcher", signalé seulement à Abbéché (Tchad), est proche du précédent. Sans subir aucune modification formelle, il exprime parfois l'imminence en remplacement de l'adverbe geri $\bar{b}$ "presque":

Abbéché, Tchad (Roth 1979: 58)

(11) ad-dūd garrab bumūt

"Le lion fut bien près de mourir"

\subsection{En berbère}

Dans les parlers berbères, peu de verbes de déplacement ont été relevés comme source lexicale du futur.

a) Suivi de $a d+$ AOR, le verbe $d d u$ "marcher" tend à exprimer le futur immédiat. C'est le cas en kabyle notamment si $d d u$ est à l'inaccompli.

Kabyle, Algérie (Naït-Zerrad 2001: 118)

(12) iteddu ad iruḥ ger lhiğ $\breve{g} \quad$ "Il était sur le point d'aller faire le pélerinage"

En chleuh, le phénomène de grammaticalisation semble plus avancé car la forme conjuguée de $d d u$ (à l'accompli) peut désormais être remplacée, dans cet emploi d'auxiliaire, par la forme invariable $d d(a d)$ ( $v$. Leguil 1982):

(13) ddig ad ddug > ddad ddug $\quad$ "Je vais aller".

Le chleuh - ou du moins certaines de ses variétés - s'est donc donné un second futur: $d d a d$ s'oppose à rad comme un futur proche (plus ou moins certain) à un futur plus général ( $v$. Chaker idem: 113-4).

b) Certains locuteurs berbérophones ont introduit dans leur langue maternelle des auxiliaires d'origine arabe. Ainsi les Senhaja de Srär emploient-ils le māšsi arabe, au lieu du g $a$ de leurs voisins rifains ( $v$. Renisio 1932: 49).

(14) māši ađ yaf

"Il va trouver, il trouvera, il doit trouver"

Il y a eu emprunt, mais la présence de $a d$ (avec $d$ spirantisé: $đ$ ) devant le verbe principal montre que la construction n'est pas complètement décalquée de l'arabe. 


\section{DES CAS PARTICULIERS DE VISEE}

La visée joue un rôle important dans la grammaticalisation des morphèmes de futur, non pas qu'il soit inhabituel de « viser » le passé ou le présent, mais parce que le temps du futur est, plus que les autres, celui des événements non (encore) advenus qui ne se laissent représenter que mentalement. Si elle est consubstantielle à la notion de désir, la visée n'est pas étrangère non plus à celle de mouvement, pour peu que celui-ci soit considéré comme un mouvement vers un but. Pourtant, dans les cas étudiés maintenant, l'expression du désir ou du mouvement tend à disparaître. L'étymon garde un lien avec la visée, mais révèle un recentrage sur l'objectif visé ou une réinterprétation sémantique de la visée.

\subsection{En arabe}

Plusieurs morphèmes de futur ont pour origine, soit une tournure exhortative, soit une particule indiquant un but ou une limite à atteindre. On verra combien ces cas peuvent être proches dans la langue.

a) L'impératif, l'hortatif et l'optatif, en tant que sources de morphème de futur, appartiennent à un type particulier, mais bien connu, de modalité orientée vers $l^{\prime}$ (inter)locuteur («speaker-oriented »vs «agent-oriented », v. Bybee \& al. idem: 23). En arabe, c'est la forme impérative du verbe xalla (en maltais halli) "laisser" qui, employée dans une construction à valeur exhortative (xalli nš̄ûf $>$ xan-nšü $f$ "laisse-moi voir !") semble à l'origine de l'expression du futur dans plusieurs parlers.

Dans le parler de l'est du Soudan, la grammaticalisation est assez poussée et la forme réduite al-/hal- ( $>$ an- à la $1 \mathrm{SG}$ et $1 \mathrm{PL}$ ) est devenue le morphème régulier du désidératif, voire du futur, dans des énoncés à intonation exclamative, ex. annašrab "ich will trinken !" (Reichmuth 1983: 290). À Malte, par contre, l'intonation est variable et généralement déterminante: en effet, les tournures avec ha $(<$ halli) prennent généralement, selon l'intonation, une valeur d'injonction ou de futur, sauf dans le cas des premières personnes (SG et PL) où elles peuvent rester ambiguës (Vanhove 2003: 153-6).

b) Avec les subjonctions finales, l'accent est mis par contre sur l'objectif visé, le but introduit par le fonctionnel (préposition ou conjonction). Plusieurs morphèmes de futur semblent trouver là leur origine. Le cas le plus connu est celui de la particule tunisienne $b \bar{a} \check{s}$ dont l'emploi comme particule de futur semble, sinon dérivé de $b \bar{a} \check{s}$ "pour que, afin que", du moins influencé par elle ${ }^{6}$. Le rapprochement avec la conjonction est conforté par le fait que biəš, l'équivalent maltais de $b \bar{a} \breve{s}$, est employé régulièrement pour exprimer l'imminence dans le passé. Il s'agit toutefois d'un emploi particulier car biəš est toujours précédé (sauf dans la variété régionale de Gozo) du verbe wasal "arriver" à l'accompli (Vanhove ibid.: 157-8).

${ }^{6}$ On peut voir en $b \bar{a} \check{s}$ une variante de la forme verbale $m \bar{a} \check{s}(i)$, mais il y a eu au moins un phénomène d'attraction et de convergence entre les deux morphèmes (Cohen 1975: 258). 
c) Au Moyen-Orient septentrional, une particule ta- $(<$ hatta(y) "afin que, pour que, jusqu'à ce que") accompagne régulièrement l'inaccompli « nu » dans différents emplois de non factuel, avec des valeurs diverses ${ }^{7}$.

- Dans certains parlers (libanais notamment), l'emploi de ta est celui d'un subordonnant, même s'il sert essentiellement « à marquer l'intention de l'agent, et indiquer l'objet ou le résultat de l'action » (Feghali 1928: 83).

- Dans d'autres parlers, $t a-$, particule habituelle de l'exhortatif, fournit occasionnellement une expression de futur «immédiat», ainsi à Qašqa-daryā : ta-nogdi "Let's go, we'll go now, we're going already" (Chikovani 2002: 186).

- Mais en Anatolie et au Kurdistan - dans les deux branches Nord des dialectes dits qaltu - , la particule $t a$ - (ou ses variantes to- et $d \partial$-) est devenue la marque régulière du futur. Comme ces parlers ont généralement conservé les fonctions précédentes de $t a$ - (de subjonctif aussi bien que d'optatif et exhortatif), la particule se trouve cumuler trois fonctions a priori bien distinctes.

- Enfin, dans l'arabe chypriote, s'est produit une sorte de «dédoublement » expressif du morphème $t a$ (semble-t-il sous l'influence du grec, cf. Cohen 1973: 242). ta a conservé les emplois modaux (comme dans l'ensemble syro-libanopalestinien voisin) et un morphème spécifique de futur est apparu (tta).

\subsection{En berbère}

Kabyle (Naït-Zerrad 2001: 110-111)

(15) win ara yeswen ad yehlu "Celui qui boira sera guéri"

(16) ara iru mi ara isel "Comme il pleurera quand il sera au courant"

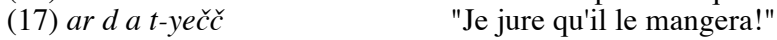

Dans les relatives (et, plus généralement, en contexte d'«attraction »), la particule $a d$ de futur est remplacée par diverses particules: kabyle ara/agia $(v$. (15) et (16)), Figuig ala, Rif $\dot{g} a$, etc. Il est notable que ces éléments se retrouvent souvent, soit dans les énoncés de serment ( $v$. Bentolila 1988), soit dans les énoncés injonctifs et optatifs négatifs (ainsi $q a$-wr en chleuh et $\dot{g} a$-wer en tamazight). Ceci semble indiquer que ces particules, à l'origine hypothétique, ont une valeur fortement expressive, peut-être essentiellement assévérative.

$\mathrm{Vu}$ le rôle particulier que les berbérisants attribuent aux relatives dans les phénomènes de grammaticalisation, que ce soit pour la formation des conjonctions (Bentolila 1981: 282 note 46, Galand 1988: 220) ou pour celle du futur (Leguil 1982), il se pourrait que le ara kabyle ait la même origine que le rad chleuh $^{8}$. Il n'est pas impossible toutefois que le fonctionnel "jusqu'à" ait joué, en berbère, un rôle comparable à celui du "jusqu'à" arabe, à ceci près qu'il aurait « renforcé » (plutôt que « remplacé ») la particule $a d$.

\footnotetext{
${ }^{7}$ Il est à noter que hatta(y) "jusqu'à (ce que)" pourrait être à l'origine, non seulement de ta, mais de plusieurs autres morphèmes de futur, tel le morphème ha- usité en Tunisie.

${ }^{8}$ Cette hypothèse semble avoir la faveur de Lionel Galand (communication personnelle), même s'il observe que le rapprochement avec la particule négative post-verbale ara (proposé dans Mettouchi 2001) constitue aussi une explication possible.
} 
En effet, la préposition ar "jusqu'à" (spatial ou temporel) semble à l'origine du préverbe ar d'inaccompli ( $v$. Chaker idem: 110-1) comme de la particule ar de serment $(v$. ex. (17)), mais elle pourrait aussi être à l'origine de la particule de futur ara attestée dans les relatives du kabyle (comme de sa variante figuiguienne ala, "jusqu'à" se disant justement al an à Figuig). La valeur assévérative acquise par les particules ar/al dans les énoncés modaux donne un supplément de vraisemblance à l'hypothèse. On peut comprendre en effet que ad seul (qui ne fait qu'actualiser l'aoriste sur l'axe du possible, $v$. Taine-Cheikh, à paraître) ne permette pas de situer temporellement un procès si l'énoncé est modal (cas des exclamatives) ou la proposition, dépendante (cas des relatives).

\subsection{En arabe et berbère mauritaniens}

Dans les parlers de Mauritanie, on a un type particulier de visée - sans volition - qui passe par des formes verbales exprimant une notion d'occupation (voire de préoccupation). En berbère zénaga, le verbe auxiliaire yänhäyä (ex. yänhäyä yəttäššäd-däh "il viendra") est, parallèlement, employé comme verbe de plein statut avec le sens de "être occupé". En arabe hassāniyya, c'est la forme invariable lāhi qui fournit l'expression régulière du futur (lāhi iži "il viendra"). Il s'agit du participe d'un verbe lhä signifiant actuellement "distraire", dont la forme dérivée lthä signifie "se distraire, s'occuper à".

Contrairement à la forme d'inaccompli du zénaga, la forme participiale du ḩassāniyya apporte une nuance de présent duratif qui rend a priori surprenante la valeur acquise par le morphème grammaticalisé. Pour sa part, Marcel Cohen (1924: 221) rapprochait lāhi de lāti qui, à Alger juif, était employé pour l'expression de la durée (lāti yākal "il est en train de manger"). Il attirait l'attention sur la possibilité d'un glissement de sens et d'emploi, de l'expression de la durée (ou de la concomitance) à celle de l'imminence. Ce glissement est difficile à expliquer, sauf à supposer qu'il a pu être favorisé par l'existence préalable de yänhäyä en zénaga9 ${ }^{9}$.

\section{DES CAS PARTICULIERS DE REPERAGE}

Le participe actif de l'arabe a une valeur expressive qui prend différentes nuances, selon l'aspect lexical du verbe («Aktionart »). Nous avons vu le cas des verbes de mouvement pour lesquels le participe, qui exprimait la phase préparatoire du procès, servait souvent de source à la forme grammaticalisée. Le lāhi mauritanien représente un autre cas de distorsion temporelle, compatible toutefois avec l'effet de visée (ou projection mentale). Les faits de grammaticalisation que nous allons examiner maintenant illustrent plus nettement encore le glissement Présent $\rightarrow$ Futur.

${ }^{9}$ On pourrait penser que yänhäyä, forme verbale toujours conjuguée, est plus récent que lāhi. Pourtant, le zénaga aurait certainement emprunté la forme au hassāniyyya plutôt qu'à l'arabe classique si l'emploi de yänhäyä était récent et calqué sur celui de l'arabe dialectal. 


\subsection{Des expressions temporelles}

Il n'est pas rare, dans les langues du monde, que des morphèmes de futur trouvent leur source dans des adverbes ou des expressions temporelles: «Adverbes indicating a time after the moment of speech or a reference time, e.g. soon, then » (Bybee \& al. 1991: 19). Dans les langues qui nous concernent, c'est un cas de figure attesté, mais peu fréquent.

a) En arabe, l'exemple le plus clair est celui du circonstant hassā̧a "maintenant" (lit. "ce-le-temps"). La variante iraquienne hassas/hassa est considérée comme la source de la particule de futur relevée dans certains parlers juifs d'Iraq, dont les réalisations sont hassas chez les Karaïtes de Hît (Khan 1997: 92), hassa et surtout sa-/ssa- à Baghdad (Blanc 1964: 117-8) ${ }^{10}$.

b) En berbère, on a l'exemple de la particule za (zé, hé) du touareg qui remplace $a d$ dans les relatives. Cet emploi de $z a$ s'éclaire grâce au rapprochement avec l'adverbe temporel $z a$ "alors, donc" attesté en chleuh (Chaker idem: 108).

\subsection{Des expressions existentielles}

a) Pour certains auxiliaires verbaux, la référence au présent semble portée par la forme aspecto-temporelle choisie. Considérons, en arabe, le verbe d'existence $k \bar{a} n(a)$ "être" qui est à l'origine de diverses particules aspecto-temporelles. Certaines grammaticalisations paraissent faciles à expliquer, ainsi l'expression du présent concomitant avec $k \bar{a}$ - (dérivé du participe $k \bar{a} y i n$ ) en Algérie. D'autres sont moins évidentes, tel kay (<kāyin) comme marque du futur en Asie centrale.

Arabe de Bukhara (Chikovani $2002: 181$ )

(18) waladak kay - miqum

"Your boy will get up"

Pour que la forme tronquée de kāyin devienne le morphème du futur, il a sans doute fallu que la valeur fortement expressive du participe présent (sa valeur première, $v$. Cohen 1984: 271) se développe, non dans le sens temporel du concomitant, mais dans celui, modal de l'inférence ${ }^{11}$.

Le glissement Présent $\rightarrow$ Futur a été observé aussi pour la forme tronquée $\$ a$ du verbe $\varsigma \bar{a} d$ "devenir; retourner" (verbe qui a donné aussi les adverbes "encore", "déjà"). Suivi d'un inaccompli, $\{a$ - est en effet attesté au Yémen avec deux valeurs distinctes: parfois il dénote le présent; souvent, comme à Yašīs et Iryān, il dénote le futur par opposition à l'action qui commence, exprimée par bi $(i)$ - ou $\bar{d}$ (cf. Diem 1973; Piamenta 1990: 314). Ce glissement à l'intérieur de ce que l'on peut appeler le «non constatif » (par opposition au passé) pourrait avoir été favorisé par la valeur d'éventualité souvent présente dans $\varsigma \bar{a} d^{12}$.

b) Le parler berbère des Aït Seghrouchen a développé, à côté du futur général

${ }^{10}$ L'élision de $h$ est normale dans certaines conditions syllabiques (Blanc idem: 139).

${ }^{11}$ Sur la valeur d'«evidential » prise en particulier par le participe présent des auxiliaires dans l'arabe du Levant $v$. Henkin 1992: 438 et $s q$.).

${ }^{12}$ La particule de futur $\uparrow a-(<\varsigma \bar{a} d)$ a également été signalée au Maghreb (ainsi à Tozeur). 
Les morphèmes de futur en arabe et en berbère

en $a d$, un second futur en $d$-ad. Celui-ci sert, soit à lever une ambiguïté ( $v$. 19b/19a), soit à apporter une nuance de certitude (v. 20b/20a $)^{13}$.

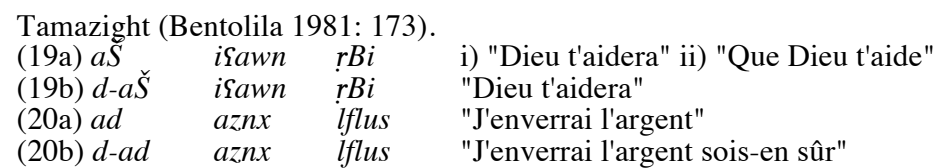

Compte tenu de la valeur du $d$ précédant la particule modale ( $d$ rajoute un accent, une emphase qui peut se rendre en français par "vraiment, justement, précisément, bel et bien, sans aucun doute, etc." », Bentolila y reconnaît le présentatif d'identification $d$ "c'est" que l'on trouve dans les énoncés nominaux du type $d$ aryaz "c'est un homme" (idem: 173, note 156).

\subsection{Des expressions d'origine déictique}

La distinction entre des futurs plus ou moins certains est une question complexe qui ne peut pas être traitée ici en détail, même si elle s'impose parfois, comme nous venons de le voir, lorsque deux formes en concurrence présentent des emplois bien différenciés.

L'arabe classique présente incontestablement plusieurs modes d'expression du futur. Outre le recours au préverbe sawfa (ou à sa variante tronquée sa-) pour l'expression du «vrai » futur, la langue ancienne avait un emploi fréquent de la conjugaison suffixale pour situer dans le futur des événements considérés comme inéluctables ( $v$. Cohen 1989: 189). Ces emplois particuliers de l'accompli existent encore dans une partie du domaine arabe et berbère, du moins dans certaines conditions syntaxiques (pour les promesses et les souhaits notamment). Des emplois de la conjugaison préfixale en - $a$ (subjonctif), peut-être plus intéressants pour notre propos, semblent par contre spécifiques à l'arabe littéraire. Ce sont, d'une part le suffixe énergique en - $n(n)$-, d'autre part la négation lan. Dans les deux cas, la présence d'un $n$ semble apporter une nuance de certitude à ces constructions.

Dans la mesure où l'hypothèse d'une origine déictique a été faite, au moins pour l'un des deux $n$ ( $v$. Zewi 2007: 22-4), le rapprochement avec les faits berbères (non seulement de $a d$, mais aussi de $d$-ad), loin de paraître complètement hasardeux, mériterait d'être approfondi.

\section{POUR CONCLURE}

Les phénomèmes de convergences n'empêchent nullement la diversité. On

${ }_{13}$ À noter que $d$-ad remplace obligatoirement $a d$ après $m a$ "est-ce que?", is "que", $m r$ "si", $z G^{w}$ is "depuis que", ani "parce que" ainsi que le coordonnant la ... la. 
aura remarqué qu'une même source lexicale avait parfois été choisie d'une extrêmité du domaine à l'autre - plus souvent cependant une source identique (un synonyme plus ou moins parfait) - , mais que les stades de grammaticalisation (figement, troncation, altération vocalique, ...) pouvaient aussi varier considérablement, même entre dialectes proches. On comprend donc que, si ces faits offrent une bonne illustration des tendances universelles en linguistique, la prise en compte de toutes ces données peut servir aussi pour classer les dialectes et déterminer des aires géolinguistiques plus ou étendues (pour l'arabe, $v$. Taine-Cheikh 2004). L'étude en parallèle des variétés arabes et berbères a par ailleurs permis de mettre en évidence, au-delà des ressemblances structurelles relativement importantes, quelques rares faits d'emprunt ou (dans le cas particulier de la Mauritanie) d'innovation convergente.

L'existence de deux niveaux de spécialisation dans les formes d'expression du futur (un niveau de «connotation » et celui de « dénotation », sur lequel nous avons concentré notre attention) repose sur le lien naturel des valeurs de futur avec l'aspect inaccompli d'une part, avec le domaine du modal d'autre part. Elle a, entre autres conséquences, celle de souligner le rôle important des constructions syntaxiques dans les phénomènes de polygrammaticalisation. Elle a de plus l'intérêt de montrer que, comme sur le plan aspectuel, les formes les plus spécialisées sont bien souvent aussi les plus lourdes, l'histoire des formes se faisant (au moins dans une grande partie du chamito-sémitique) par la marginalisation de formes anciennement marquées au profit de nouvelles, plus « emphatiques », dans un mouvement ininterrompu vers une expressivité toujours plus grande. Au plan temporel, la particularité est cependant que, si les auxiliaires d'origine verbale sont toujours aussi nombreux et si le recours au participe reste déterminant pour l'arabe, le rôle de la deixis - hérité plutôt du domaine modal - prend une place plus importante et devient (presque clairement) identifiable. Que les marques de futur trouvent leur source métaphoriquement dans un mouvement (généralement celui du corps ou de l'esprit de l'énonciateur) ou qu'elles naissent de l'expression de la quasi-concomitance avec le moment de l'énonciation, il paraît souvent bien difficile d'effectuer une coupure nette entre futur et présent d'une part, futur et modal d'autre part.

\section{Références}

Behnstedt P., 1997. Sprachatlas von Syrien. I: Atlas, Wiesbaden: Harrassowitz. Bentolila F., 1981. Grammaire fonctionnelle d'un parler berbère: Aït Seghrouchen d'Oum Jeniba (Maroc), Paris: SELAF.

Bentolila F., 1988. Les syntagmes verbaux des serments dans différents parlers berbères, Awal 4, p. 43-72.

Blanc H., 1964. Communal Dialects in Baghdad, Cambridge: Harvard U.P. 
Brugnatelli V., 2005. Un nuovo poemetto berbero ibadita, in Studi Magrebini (N.S.) 3 (Studi Berberi e Mediterranei), Napoli, p. 131-142.

Bybee J.L., W. Pagliuca \& R.D. Perkins, 1991. Back to the Future, in Traugott E.C. \& Heine B. (eds), Approaches to grammaticalization, Amsterdam/ Philadelphia: Benjamins Publishing Company, p. 17-58.

Chaker S., 1997. Quelques faits de grammaticalisation dans le système verbal berbère, in Grammaticalisation et reconstruction, Mémoires de la SLP, Paris: Klincksieck, p. 103-121.

Chikovani G., 2002. The Verb in the Arabic Dialects of Central Asia, in Youssi A., Benjelloun F., Dahbi M. \& Iraqui-Zinaceur Z. (eds), Aspects of the Dialects of Arabic Today, Marrakesh, Rabat: Amapatril, p. 179-188.

Cohen D., 1973. Variantes, variétés dialectales et contacts linguistiques en domaine arabe, BSL LXVIII-1, p. 215-248.

Cohen D., 1975. Le parler arabe des Juifs de Tunis II. Etude linguistique. The Hague/Paris: Mouton.

Cohen D., 1984. La phrase nominale et l'évolution du système verbal en sémitique. Etudes de syntaxe historique, Paris: SLP.

Cohen D., 1988. Le chamito-sémitique, in J. Perrot (éd.), Les langues dans le monde ancien et moderne. III Les langues chamito-sémitiques, Paris: CNRS, p. 9-30.

Cohen D., 1989. L'aspect verbal, Paris: PUF.

Cohen M., 1924. Le système verbal sémitique et l'expression du temps. Paris: Leroux.

Dahl Ö. 1985. Tense and Aspect Systems, Oxford: Basil Blackwell.

Diem W., 1973. Skizzen jemenitischer Dialekte, Beirut/Wiesbaden: Steiner.

Féghali Mgr M. 1928. Syntaxe des parlers arabes actuels du Liban, Paris: Geuthner.

Galand L., 1977. Continuité et renouvellement d'un système verbal: le cas du berbère, BSL LXXII-1, p. 275-303.

Galand L., 1988. Le berbère, in J. Perrot (éd.), Les langues dans le monde ancien et moderne. III Les langues chamito-sémitiques, Paris: CNRS, p. 207-242.

Gosselin L, 1996. Sémantique de la temporalité en français. Un modèle calculatoire et cognitif du temps et de l'aspect, Louvain: Duculot.

Heine B. \& Kuteva T., 2002. World Lexicon of Grammaticalization, Cambridge: Cambridge University Press.

Henkin R., 1992. The three faces of the Arabic participle in Negev Bedouin Dialect : continous, resultative and existential, BSOAS LV-3, p. 433-46.

Khan G., 1997. The Arabic Dialect of the Karaite Jews of Hît, Zeitschrift für arabische Linguistik 34, Wiesbaden, p. 53-102.

Klein E., 2003. Les tactiques de Chronos, Paris: Flammarion.

Kossmann M., 1997. Grammaire du parler berbère de Figuig (Maroc oriental), Paris-Louvain: Peeters.

Kossmann M., 2000. Esquisse grammaticale du rifain oriental, Paris-Louvain: Peeters.

Laoust E., 1931. Siwa. Son parler, Paris: Leroux.

Leguil A., 1982. La naissance des temps en chleuh, Bull. des Etudes africaines de l'Inalco 3, Paris, p. 57-84.

Marçais Ph., 1956. Le parler arabe de Djidjelli (Nord Constantinois, Algérie), Paris: Maisonneuve.

Mettouchi A., 2001. La grammaticalisation de ara en kabyle, négation et subordination relative, Travaux du CerLiCO 14, p. 215-235. 
Naït-Zerrad K., 2001. Grammaire moderne du kabyle. tajerrumt tatrart $n$ teqbaylit, Paris: Karthala.

Ouhalla J., 2008. Negation, in K. Versteegh (éd.), Encyclopedia of Arabic Language and Linguistics III, Leiden: Brill, p. 355-60.

Piamenta M., 1990. Dictionary of Post-Classical Yemeni Arabic, Leiden/New York/Kābenhavn/Köln: Brill.

Procházka S., 2003. The Bedouin Arabic dialects of Urfa, in Ferrando F. \& Sanchez Sandoval J. J. (eds), 2003. AIDA 5th Conference Proceedings, Cadiz september 2002, Cadiz: Universidad de Cadiz, p. 75-88.

Reichmuth S., 1983. Der arabische Dialekt der Škriyya im Ostsudan, Hildesheim/Zürich/New York: Olms.

Renisio A., 1932. Etude sur les dialectes berbères des Beni Iznassen, du Rif et des Senhaja de Srä̈r, Paris: Leroux.

Rosenhouse J., 1981. Some Features of some Bedouin Dialects in the North of Israel , Zeitschrift für arabische Linguistik 7, Wiesbaden, p. 23-47.

Rossi E., 1938. Appunti di dialettologia del Yemen, Rivista degli studi orientali 17, Rome, p. 230-65

Roth A., 1979. Esquisse grammaticale du parler arabe d'Abbéché (Tchad), Paris: Geuthner.

Taine-Cheikh C., 2004. Le(s) futur(s) en arabe. Réflexions pour une typologie, Estudios de dialectología norteafricana y andalusí 8, Zaragoza, p. 215-238.

Taine-Cheikh C., à paraître. The role of the Berber deictic ad and TAM markers in dependent clauses in Zenaga. in I. Bril (éd.) Clause-hierarchy and Clauselinking: the Syntax and pragmatic interface. Amsterdam/ Philadelphia: J. Benjamins Publishing Company.

Vanhove M., 2003. Auxiliaires, grammaticalisation et transcatégorialité: le cas des marqueurs de futur et d'injonction en maltais, in S. Robert (éd.), Perspectives synchroniques sur la grammaticalisation, Louvain/Paris: Peeters, p. 149-162.

Zewi T., 2007. Energicus, in K. Versteegh (éd.), Encyclopedia of Arabic Language and Linguistics II, Leiden: Brill, p. 22-25. 